\title{
Diffraction and forward physics results of the ATLAS experiment from the Run I
}

\author{
Marek Taševský, on behalf of the ATLAS Collaboration \\ Institute of Physics, Academy of Sciences of the Czech republic, Na Slovance 2, 18221 Prague, Czech republic
}

\begin{abstract}
Various aspects of forward physics have been studied by the ATLAS collaboration using data from Run I at the LHC. In this text, main results of four published analyses are summarized, all based on data from proton-proton collisions at $\sqrt{s}=7 \mathrm{TeV}$ collected in 2010 or 2011 . Two analyses deal with the diffractive signature, one based on single-sided events, the other on large rapidity gaps in soft events. In addition, a recent measurement of the total pp cross section using the ALFA subdetector and a recent study of higher-order QCD effects using a jet veto are discussed.
\end{abstract}

Keywords: elastic scattering; inelastic scattering, single diffraction, rapidity gap, higher-order QCD; jet azimuthal decorrelations PACS: $13.85 . \mathrm{Hd}$

\section{INTRODUCTION}

The diffractive analyses, one based on single-sided events and estimating the total inelastic cross section [1], the other providing rapidity gap distribution compared with diffractive and hadronization models [2], were already discussed in $[3,4]$ and here only basic facts are reminded. More emphasis is put on recent measurements with the ALFA subdetector [5] and studies using the jet veto and jet azimuthal decorrelations [6]. Larger gaps between particles can be due to statistical fluctuations in the hadronization process, which are exponentially suppressed [7] as a function of gap size, or by $t$-channel color singlet exchange (CSE) which can be provided by electroweak or more frequently by strongly interacting states. The latter processes are termed as diffractive and their phenomenological description is inspired by Regge theory where the CSE is associated with the Pomeron [8, 9]. Inclusive inelastic (hereafter called Minimum Bias (MB)) processes are formed by two classes, the non-diffractive (ND) and diffractive processes, where the latter is divided further according to where the gap is found, to Single dissociation (SD) (one gap close to the intact leading proton), Double dissociation (DD) (one gap in the central rapidity region) or Central diffraction (CD) (both intact leading protons accompanied by one gap). The kinematics of the diffractive dissociation is commonly described in terms of the four-momentum transfer squared, $t$, and momentum fraction loss of the intact leading proton, $\xi$, calculated as $M^{2} / s$ where $M$ is the invariant mass of the dissociated system and $s$ is the squared center-of-mass energy. The gap (i.e. the pseudorapidity difference) between the intact proton and the dissociated system is related to $\xi$ by the relation $\Delta \eta \simeq-\ln \xi$. The pseudorapidity is defined as $\eta=-\ln \tan (\theta / 2)$ where $\theta$ is the polar angle with respect to the incoming beam direction. Prospects for the diffractive program in the ATLAS experiment at the LHC were outlined e.g. in [10].

\section{SINGLE-SIDED EVENTS}

Diffractive signal in an event sample can be enhanced by requiring no activity (above a noise threshold) in a part of the detector. The MB events are selected using the Minimum Bias Trigger Scintillator (MBTS) consisting of two discs symmetrically placed $\pm 3.56 \mathrm{~m}$ away from the interaction point (IP) and covering $2.1<|\eta|<3.8$. The trigger efficiency is larger than $99 \%$ for particles produced in this $\eta$ region. In the analysis [1] the gap is looked for by vetoing the activity in the MBTS on one side of IP and requiring at least one track in the inner tracking detector (ID) with $p_{T}>0.5 \mathrm{GeV}$. The measured ratio of such single-sided to all events, $R_{S S}=(10.0 \pm 0.4) \%$, when compared to various models, requires the fraction of diffractive to all inelastic processes to be $26.9_{-1.0}^{+2.5} \%$ depending on the model. The systematic uncertainties come from varying a DD/SD event ratio between 0.0 and 1.0 using the default DonnachieLandshoff model (DL) of diffractive scattering [11] and keeping it consistent with data $R_{S S} \pm 1 \sigma$ value. The total inelastic cross section measured in the accessible region $\xi>5 \times 10^{-6}$ is $\sigma_{\text {inel }}=60.33 \pm 2.10 \mathrm{mb}$. Extrapolating it to the full $\xi$-range using the DL model gives $\sigma_{\text {inel }}=69.4 \pm 2.4 \pm 6.9$ (extrap.) mb. 

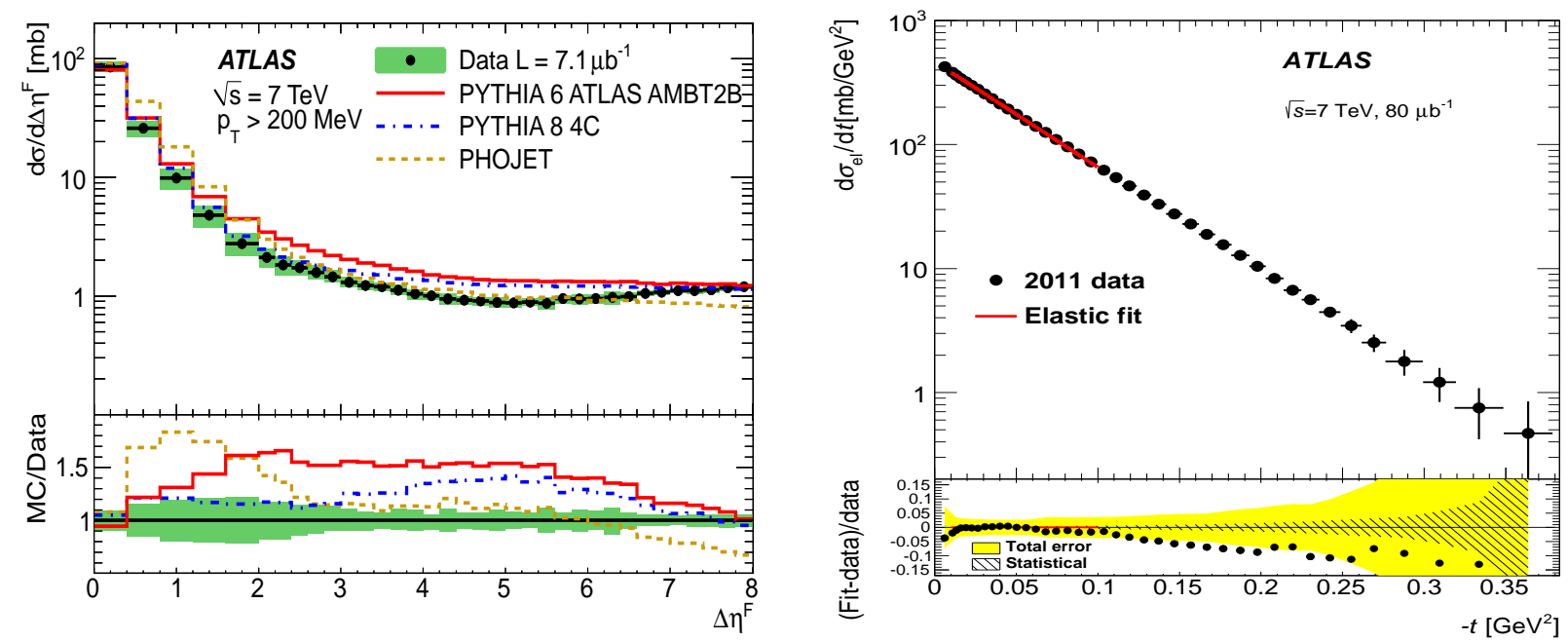

FIGURE 1. Left: Inelastic cross section differential in forward gap size for particles with $p_{T}>200 \mathrm{MeV}$. The shaded bands represent the total uncertainties in the data. Predictions of Phojet and the default versions of Pythia 6 and Pythia 8 are shown by dashed, full and dashed-dotted line, respectively. Taken from [2]. Right: Measured differential elastic cross section reconstructed with the subtraction method and its fit using theoretical prediction with $\sigma_{\text {tot }}$ and B as free parameters. In the lower panel the points represent the normalized difference between fit and data, the yellow area represents the total experimental uncertainty and the hatched area the statistical component. The red line indicates the fit range, the fit result is extrapolated in the lower panel outside the fit range. Taken from [5].

\section{RAPIDITY GAPS IN SOFT EVENTS}

Rapidity gaps in this analysis [2] were reconstructed using the calorimeter system of the ATLAS detector [12] consisting of the electromagnetic (EM) $(|\eta|<3.2)$, hadronic (H) end-cap $(1.5<|\eta|<3.2)$ and EM/H forward system $(3.1<|\eta|<4.9)$, complemented by the steel/scintillator hadronic tile calorimeter $(|\eta|<1.7)$. The calorimetric information is then combined with the information from the ID for $|\eta|<2.5$. An interval in $\eta$ is considered to be empty of final state particles if it does not contain clusters that pass noise suppression requirements for respective calorimeter subsystems and have $p_{T}>p_{T}^{\text {cut }}$ or good tracks in ID with $p_{T}>p_{T}^{\text {cut }}$. Detector simulation showed that the lowest accessible energy with a good acceptance is for clusters with $p_{T}>200 \mathrm{MeV}$. The forward gap size $\Delta \eta_{F}$ is defined by the larger of the two empty rapidity regions extending between the edges of the detector acceptance at $\eta$ $=4.9$ or -4.9 and the nearest track or calorimeter cluster passing the selection requirements at smaller $|\eta|$. Results corrected for detector effects to the level of stable particles and for $p_{T}^{c u t}=200 \mathrm{MeV}$ are shown in Fig. 1 left where the measured differential inelastic cross section $d \sigma / d \Delta \eta_{F}$ is compared to predictions of PYTHIA 6 and 8 and PHOJET. The data show an exponential decrease for $\Delta \eta_{F}<2.5$ attributed to ND processes and a plateau for $\Delta \eta_{F}>3$ typical for diffractive processes. Although all models follow these general features seen in data, they all fail to describe both the shape and normalization over the whole gap range. This is not surprising since none of these models have been tuned to describe diffractive processes at LHC and this measurement can serve this purpose. Other results obtained in this analysis are a study of dynamics, another hadronization model and the inelastic cross section as a function of $\xi$. Dynamics of observed diffractive processes was studied via fitting the slope, so called Pomeron intercept, $\alpha_{P}(0)$, of the $\Delta \eta_{F}$ distribution at large gaps. Using PYTHIA 8 with the DL model, $\alpha_{P}(0)$ was found to be $1.058 \pm 0.003$ (stat. $)_{-0.039}^{+0.034}$ (syst.) which due to large systematic uncertainties is still compatible with the default DL value 1.085 [11]. A comparison of predictions of HERWIG++ based on the cluster hadronization model in the tune MB UE7-2 [2] revealed an unexpected feature. Although not containing any explicit diffractive component, HERWIG++ produces large gaps with non-exponential behaviour and a bump at $\Delta \eta_{F}=6$. Finally, the inelastic cross section integrated over an interval $0<\Delta \eta_{F}<\Delta \eta_{F}^{\text {cut }}$ can be converted into the integral over the interval $\xi_{\text {cut }}<\xi<1$ thanks to the strong correlation $\Delta \eta \simeq-\ln \xi$. 


\section{TOTAL AND ELASTIC PP CROSS SECTION}

The total pp cross section has recently been measured in ATLAS [5] using the ALFA forward proton subdetector placed at $240 \mathrm{~m}$ from IP and via the optical theorem which relates the total cross section with the imaginary part of the amplitude for elastic processes. Results are based on data collected during a dedicated run in 2011 with $\beta^{*}=90 \mathrm{~m}$ beam optics (together with the Totem experiment) with a negligible effect of pile-up and corresponding to an integrated luminosity of $78.7 \mu b^{-1}$. Thanks to fiducial and geometrical cuts posed on tracks in ALFA to select elastic events, the remaining background was found to be below $0.5 \%$, while keeping the acceptance above $20 \%$ in most of the accessible $t$-range. Data were corrected for detector effects using an unfolding method based on a smearing matrix constructed from Pythia 8 events. Values of $t$ variable were constructed using the so called subtraction method giving the best acceptance and resolution, while checked with other, less performant, methods. Many other systematic checks are reflected in the total systematic uncertainty and include differences between nominal and alternative methods used in the alignment and unfolding procedures and in calculating e.g. integrated luminosity, background, beam optics parameters, event reconstruction and tracking efficiency and others. The largest systematic uncertainty comes from the luminosity measurement. The values $\sigma_{\mathrm{tot}}=95.35 \pm 1.36 \mathrm{mb}$ and $B=19.73 \pm 0.24 \mathrm{GeV}^{-2}$ for the nuclear slope have been obtained by fitting the elastic cross section differential in $t$ in the range $t \in<0.01,0.1>\mathrm{GeV}^{-2}$ and using a theoretical formula containing terms for Coulomb and nuclear interactions, and for Coulomb-nuclear interference and leaving $\sigma_{\text {tot }}$ and $B$ as free parameters. Assuming the nuclear term only and constant $B$, the total elastic cross section has been extracted to be $\sigma_{\mathrm{el}}=24.00 \pm 0.60 \mathrm{mb}$ and the total inelastic cross section $\sigma_{\text {inel }}=71.34 \pm 0.90 \mathrm{mb}$. All cross section measurements are in very good agreement with the Totem results [13] (differences slightly above $1 \sigma$ ) with ATLAS reaching the total systematic uncertainty by $40 \%$ smaller compared to those reported by Totem. This difference is attributed to the difference between the luminosity measurement uncertainties (2.3\% in ATLAS and $4 \%$ in CMS). The precision of the ATLAS measurement of the inelastic cross section has been significantly improved compared to the results obtained in the previous measurement (described above).

\section{JET VETO}

The jet veto is a method used to define gaps in detector. First ATLAS results based on 2010 data amounting to an integrated luminosity of $36 \mu \mathrm{b}^{-1}$ were published in [14]. In inclusive dijet events, i.e. in events with at least two jets with $p_{\mathrm{T}, \mathrm{j} 1}, p_{\mathrm{T}, \mathrm{j} 2}, y_{\mathrm{j} 1}$ and $y_{\mathrm{j} 2}$ denoting transverse momenta and rapidities of two jets with highest $p_{\mathrm{T}}$ (defining the dijet system), the gap event is identified if no additional jet in the rapidity interval bounded by the dijet subsystem (defining the jet separation $\Delta y=y_{\mathrm{j} 1}-y_{\mathrm{j} 2}$ ) with a scale larger than a veto scale $Q_{0}$ is found. We define the gap fraction by a ratio of numbers of the gap events to all inclusive dijet events. A new analysis [6] includes all 2010 data and uses an optimized 2-jet trigger procedure to reach $\Delta y$ of up to 8 (in 2010 data where the pile-up effect is low) and adds the 2011 data $\left(4.5 \mathrm{fb}^{-1}\right)$ to extend the high- $p_{\mathrm{T}}$ region up to $1.5 \mathrm{TeV}$. Jets were found by the anti-kt algorithm with radius $R=0.6$ and have to pass the following criteria: $p_{\mathrm{T}, \mathrm{j} 1}>60 \mathrm{GeV}, p_{\mathrm{T}, \mathrm{j} 2}>50 \mathrm{GeV},\left|y_{\mathrm{j} 1 \mathrm{j} 2}\right|<4.4$ and $Q_{0}=p_{\mathrm{T}}>20(30) \mathrm{GeV}$ for 2010 (2011) data.

The goal of this analysis is to study higher-order (HO) QCD effects in the dijet production. These are most visible when in the dijet system i) jet separation, $\Delta y$, is large or ii) jet veto between the jets (with scale $Q_{0}$ ) is applied or iii) azimuthal decorrelation between the jets, $\pi-\Delta \phi$, is large. In theoretical predictions, the HO effects are resumed in terms of $\ln \left(Q^{2}\right)$ where $Q^{2}$ is a hard scale of the interaction (DGLAP approach) or in terms of $\ln (1 / x)$ where $x$ is Bjorken- $x$ (BFKL approach). The gap fraction is expected to be sensitive to the DGLAP effects (wide-angle soft gluon radiation) if an average $p_{\mathrm{T}}$ of the dijet system, $\overline{p_{\mathrm{T}}}$, is much larger than the veto scale, to the BFKL effects if $\Delta y$ is large and to Color-Singlet exchange if both $\overline{p_{\mathrm{T}}} / Q_{0}$ and $\Delta y$ are large. On the other hand, the azimuthal decorrelations are sensitive to both, the DGLAP and BFKL effects. It is thus natural to look at different corners of phase-space where one effect is largely dominant over the other two and try to compare with models which include the studied effect. Data are compared to predictions of two models. Powheg model calculates NLO dijet cross sections based on the DGLAP formalism and is interfaced with Pythia 8 or Herwig to resum soft and collinear emissions using parton shower approximation. The HEJ model provides leading-logarithm calculations of multi-jet (at least two hard jets) production in Mueller-Navelet limit and is interfaced to ARIADNE which contains color-dipole cascades producing soft and collinear radiation in the gap between jets and thereby giving contributions from low $x$ BFKL-like logarithmic terms. One of the most interesting results in [6] is the gap fraction as a function of $\overline{p_{\mathrm{T}}}$ and $\Delta y$. While in data a steeply falling dependence in most of the spectra is attributed to the exchange of color in the $t$-channel, plateaus observed at large values of $\overline{p_{\mathrm{T}}}$ and $\Delta y$ may be explained by i) steeply falling parton distribution functions at large $x$ disabling 

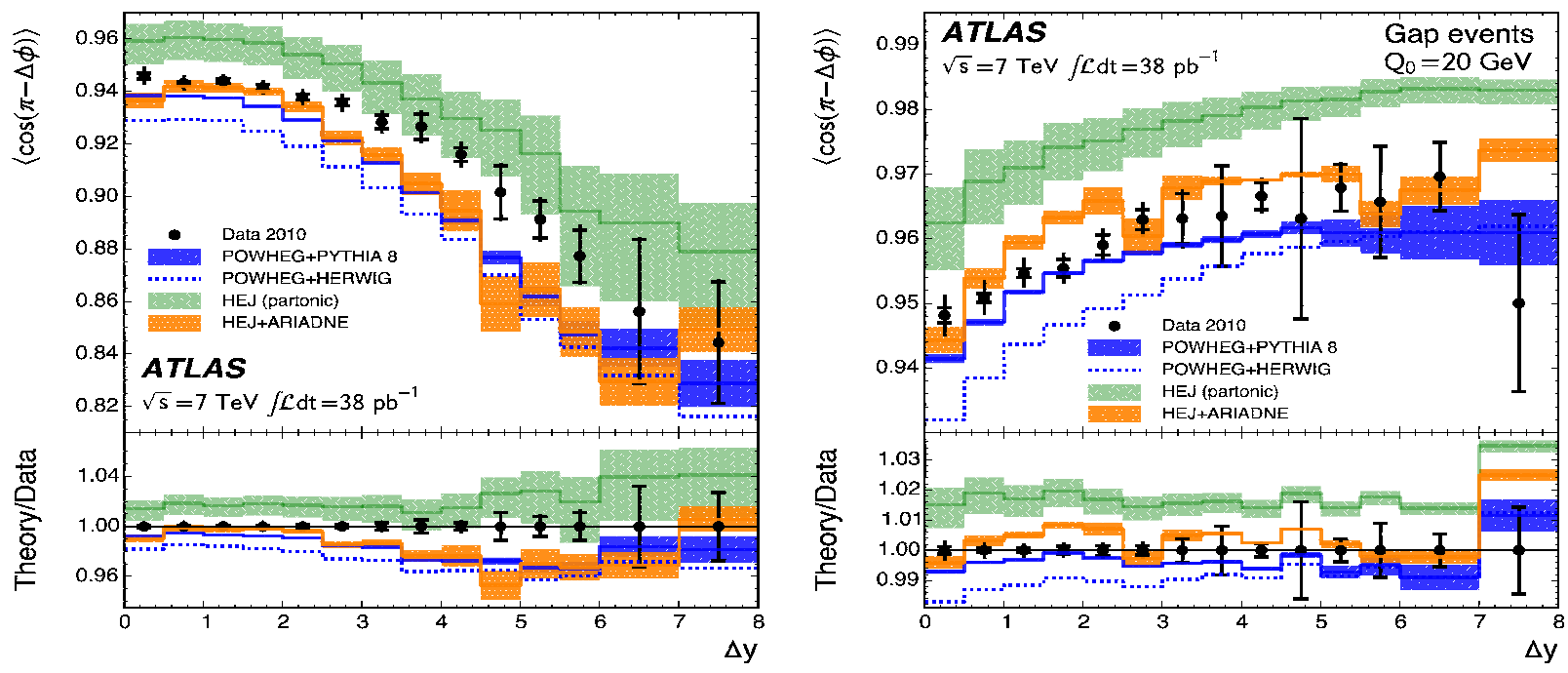

FIGURE 2. The measured $<\cos (\pi-\Delta \phi)>$ distribution as a function of $\Delta y$ for all events (Left) and gap events (Right). The veto scale is $Q_{0}=20 \mathrm{GeV}$ for data collected during 2010. The data are compared with predictions of the HEJ, HEJ+ARIADNE, POWHEG+PYTHIA and POWHEG+HERWIG models. Taken from [6].

additional parton radiation and/or ii) by contribution of CSE processes (cross section for CSE rises with increasing $\Delta y$ ). Contrary to the trend seen in data, all models show a fall at high $\Delta y$. In Fig. 2, azimuthal decorrelations are shown as a function of $\Delta y$ for inclusive dijet and gap events. We observe that while Powheg underestimates, HEJ overestimates azimuthal decorrelations observed in data. With increasing $\Delta y$ and $\overline{p_{T}}$, the jet veto makes the dijet system more backto-back. From other distributions presented in [6] such as gap fraction as a function of $Q_{0}$ for slices of $y$, jet multiplicity as functions of $\Delta y$ and $\overline{p_{\mathrm{T}}}$ or $\Delta y$ and $\Delta \phi$ distributions, one concludes that the best agreement to data is achieved with Powheg+Pythia 8 except for low $\overline{p_{\mathrm{T}}}$ and high $\Delta y$ region and that interfacing ARIADNE to HEJ significantly improves the description of data.

\section{ACKNOWLEDGMENTS}

Supported by the project LG13009 of the Ministry of Education of the Czech republic.

\section{REFERENCES}

1. ATLAS Collaboration, Nature Commun. 2 (2011) 463.

2. ATLAS Collaboration, Eur. Phys. J. C72 (2012) 1926.

3. M. Tasevsky, AIP Conf. Proc. 1350 (2011) 164.

4. M. Tasevsky, AIP Conf. Proc. 1523 (2012) 306.

5. ATLAS Collaboration, arXiv:1408.5778 [hep-ex].

6. ATLAS Collaboration, Eur. Phys. J. C74 (2014) 3117.

7. J. Bjorken, S. Brodsky, H. J. Lu, Phys. Lett. B286 (1992) 153.

8. E. Feinberg, I. Pomerancuk, Suppl. Nuovo Cimento, 3 (1952) 652.

9. G. Chew, S. Frautschi, Phys. Rev. Lett., 7 (1961) 394.

10. M. Tasevsky, Nucl. Phys. Proc. Suppl. 179-180 (2008) 187; ATL-PHYS-CONF-2008-019.

11. A. Donnachie, P. Landshoff, Nucl. Phys. B244 (1984) 322.

12. ATLAS Collaboration, J. Inst. 3 (2008) S08003.

13. G. Antchev et al. (Totem Collaboration), Eur. Phys. Lett. 101 (2013) 21002; Eur. Phys. Lett. 101 (2013) 21004; Eur. Phys. Lett. 101 (2013) 21003.

14. ATLAS Collaboration, JHEP 1109 (2011) 053. 